\author{
Ivana Simić Ćorluka* \\ Università di Belgrado
}

\title{
SESSUALITÀ E SENESCENZA IN UN CUNTO DEL BASILE**
}

\begin{abstract}
Nell'età barocca quando il compito principale dell'arte diventa la meraviglia e iniziano a venir indagate quelle realtà che nelle epoche precedenti erano state emarginate, l'identità corporea ritorna sotto la lente d'ingrandimento dell'artista, facendo emergere nella produzione artistica temi e personaggi fino ad allora quasi inesistenti. Così il Basile, in un cunto della sua opera monumentale Lo cunto de li cunti, introduce come protagoniste due vecchiette che diventano oggetto del desiderio sessuale di un re. Attraverso questo plot inconsueto, l'autore non soddisfa soltanto le bizzarrie del barocco e i suoi imperativi tematici e stilistici, ma apre questioni complesse in polemica con le epoche precedenti, offrendo la sua visione della vita, stavolta in una luce prospettica relativa, pessimista e multiforme.
\end{abstract}

Parole chiave: Basile, barocco, vecchiaia, gioco, riso, metamorfosi.

Già nel Cinquecento assistiamo a una serie di immagini iconografiche che dimostrano una spiccata sensibilità verso quei temi che alcuni decenni dopo diventeranno il leitmotiv della produzione artistica: i cicli della vita, il tempo, la fragilità del corpo, l'imperfezione umana'. Tutti questi temi,

*ivana.simic.corluka@fil.bg.ac.rs

** Dedicato alla Prof.ssa Mirka Zogović come piccolo segno di gratitudine.

${ }^{1}$ Per esempio, nel suo quadro famoso "Le tre età dell'uomo" (l'immagine è disponibile sul sito https://discpellegrina.blogspot.com/2016/01/le-tre-eta-delluomo-hans-baldung. html), Hans Baldung Grien (circa 1485-1545) raffigura un'immagine inquietante della fuggevolezza della vita incarnata da tre figure, ognuna delle quali simboleggia appunto una fase della vita: la giovinezza, la vecchiaia e la morte. Anche se il biancore della sua pelle e il suo corpo formoso contrastano con i toni giallastri degli altri due personaggi e con i loro corpi sformati, la donna giovane non può godersi la bellezza che la sua età le dona, ma accigliata, con una lacrima sul viso, cerca di salvare il drappo che la seconda figura, che impersona la vecchiaia, prova a strapparle. La Vecchia, con una faccia bieca, di una nudità angosciante, guarda in direzione della giovane, decisa a coprirsi con il suo drappo, come se, rubandoglielo, potesse prendersi anche un po' della sua giovinezza e 
pur presenti fin da prima, vengono ora privati del significato metafisico e degli ammonimenti di natura religiosa. Nel Seicento lo sguardo si concentra ancor di più sull'uomo e l'amarezza tassiana di fronte alla debolezza umana e alla sua incapacità di svincolarsi dal mondo materiale e dedicarsi a quello eterno si trasforma spesso nello sconforto provocato dalla caducità della bellezza del corpo, soprattutto quello femminile, dove mancano richiami che vadano oltre la vita terrena. Tuttavia, non sempre la riflessione sull'impossibilità di riportare il tempo indietro e, con esso, anche la bellezza e la giovinezza, assume toni melanconici e cupi. "Fare d'Ecuba un'Elena" diventa plausibile e allo stesso tempo anche dilettevole, quando Giambattista Basile (1566-1632) sotto lo pseudonimo anagrammatico di Gian Alessio Abbattutis, con il quale sottoscriverà tutte le sue opere dialettali, ci offre un processo inverso nel cunto finale della prima giornata de Lo Cunto de li cunti, «La vecchia scorticata». Quest'opera, pubblicata postuma, generosamente propone al pubblico tutti i temi cari al gusto dell'epoca barocca e, nel caso del Basile, apre un mondo che si spinge oltre il semplice e volutamente ingannevole sottotitolo - Lo trattenimiento de peccerille.

Anche se le protagoniste del cunto «La vecchia scorticata» sono entrate in quella fase della vita che quasi confina con la morte, la morte non viene nemmeno menzionata, se non alla fine, e poi arriva non come fatto naturale, ma come conseguenza dell'atto sciocco della sorella più giovane. L'unico segno tangibile della loro vecchiaia ormai avanzata è il loro aspetto fisico, la cui descrizione è portata all'estremo; per il resto, possiamo dire che le due vecchiette hanno in dono qualcosa che in maniera stereotipata viene legato esclusivamente al mondo dei giovani: la propensione al gioco e $\mathrm{i}$ "grilli per la testa".

freschezza, quasi ignorando la presenza dello scheletro che stringe in mano una clessidra, tacita ammonizione al fatto che la sua vita fra poco sarà finita. In fondo ai loro piedi dorme un bambino, accanto al quale, come se non bastassero la presenza delle "tre Grazie" e la simbologia che incarnano, si aggira un gufo, aggiungendo un ulteriore memento mori. Tutti e tre i personaggi principali sono visibilmente e essenzialmente inseparabili l'uno dall'altro (la Vecchia ha una mano posata sulla spalla della Giovane, mentre la Morte tiene la Vecchia sotto il braccio). La Vecchia ricorda alla Giovane la caducità della vita, mostrandole in che cosa si trasformeranno la sua bellezza e il suo corpo, mentre invece la Morte, tenendola strettamente sotto braccio, trascina la Vecchia con sè, dimostrando l'inesorabilità del destino.

${ }^{2}$ Luciano in un epigramma scrive: "Il crin tu tinger puoi, ma la vecchiezza / non tingerai giammai, nè dalle guance / Distenderai le rughe... / Dunque non imbrattar tutto il viso, / Che una maschera mostri, non il volto. / Perocchè nulla cià ti giova. Quale / Follia è questa? belletto, e colore / Un'Elena nommai d'Ecuba fece'. Testo disponibile sul sito https://books.google.rs/books?id=P75BOzIsooEC\&printsec $=$ frontcover\&hl=it\#v=onepa ge\&q\&f=false. 
Come all'inizio di ogni cunto, il Basile, attraverso la voce delle sue narratrici, questa volta della Iacova squarquoia ${ }^{3}$, introduce il tema del cunto nella rubrica che riassume la storia:

Lo re de Roccaforte se 'nnammora de la voce de na vecchia, e, gabbato da no dito rezocato [... e, ingannato da un dito succhiato], la fa dormire cod isso. Ma, addonatose de le rechieppe, la fa iettare pe na fenestra e, restanno appesa a n'arvolo [Ma, accortosi delle pellacce, la fa gettare dalla finestra e, quella, rimasta appesa ad un albero], è fatata da sette fate e, deventata na bellissema giovana, lo re se la piglia pe mogliere. Ma l'autra sore, 'nmediosa de la fortuna soia [invidiosa della sua fortuna], pe farese bella se fa scortecare e more (Basile 2013: 198).

Già nella rubrica troviamo un tema abbastanza bizzarro unito ad una lingua dialettale che furono, del resto, la causa principale delle molte controversie che l'opera basiliana suscitò subito dopo l'uscita, nonché di una serie di attributi come osceno, immorale, disgiunto, ecc. che tali scelte tematiche e linguistiche provocarono. Inoltre, la scelta della lingua napoletana traeva sovente i vari ricercatori (soprattutto quelli del romanticismo, come per esempio i Fratelli Grimm) in un altro inganno (Maggi 2015: 28-29) e li portava alla frettolosa conclusione che la natura dominante dell'opera basiliana fosse prevalentemente orale ${ }^{4}$.

Sull'esempio del modello decameroniano, dopo la rubrica segue l'introduzione al cunto che prende in considerazione anche il suo peso morale

${ }^{3}$ Ognuna delle narratrici incarna qualche difetto fisico, al contrario dei novellatori decameroniani che rappresentano l'incarnazione di ogni perfezione e nobiltà, sia del corpo che morale. L'epiteto che qualifica la narratrice dell'ultimo cunto della prima giornata, come osserva Picone (2003: 306-307), comunica la somiglianza tra il suo dono del racconto e la squacquera, cioè "un forte attacco di diarrea; Iacova non sa tenersi niente dentro, ma è presa dalla foga inarrestabile di raccontare tutti i racconti che conosce...".

${ }^{4}$ Questa conclusione si basava esclusivamente sull'apparenza formale dei cunti, trascurando la spiccata elaborazione retorica tipica del barocco, accompagnata dalla presenza di quattro egloghe che chiudono le prime quattro giornate, e trascurando soprattutto l'intertestualità con la quale il Basile comunicava con i suoi predecessori, il che prova la natura altamente letteraria dei cunti. Prima di tutto si pensa al Boccaccio e al suo Decameron che rimane il modello principale, e non soltanto dal punto di vista strutturale. Con il Decameron il Basile ora entra in confronto, ora lo altera, ora lo elabora, ottenendo in quel modo qualcosa che secondo Getto diventa "una specie di variazione di quel modello, la quale avviene secondo la direzione imposta dalla nuova sensibilità, una variazione che è già per se stessa frutto di quel gusto capriccioso, di quel desiderio di rottura dei vecchi schemi che contraddistingue il Barocco" (Getto 2000: 297). Questa affiliazione sarà la ragione per cui l'edizione del 1674, curata da Pompeo Sarnelli, esce con il titolo Il Pentamerone overo Lo cunto de li cunte, appellativo che molto spesso l'accompagnerà da quel momento in poi. 
e il suo significato metaforico. La narratrice pone come problematica centrale la vanità delle femmine e la loro ossessione per la bellezza che vanno condannate soprattutto se germogliano nella mente di una vecchia che vuole competere co le figluole (Basile 2013: 198). Iacova si sofferma sul tema dell'invidia, ponendola come nucleo della storia e trova una conferma della sua tesi nella citazione di Sannazzaro che fatalmente chiude l'intero cunto, "la "nmidia, figlio mio, se stessa smafara" [strugge se stessa] (Basile 2013: 216), spegnendo anche la vita di una delle due protagoniste. Eppure, sembra che il narratore extradiegetico abbia avuto in mente qualcosa che andasse molto più oltre la semplice invidia, qualcosa che trapassasse il corpo del microtesto e entrasse in comunicazione con l'opera intera, illustrando non soltanto il proprio punto di vista, ma la presa di posizione dell'intera epoca e dell'ambiente a cui apparteneva rispetto alla sensualità e alla sessualità.

Comunque, non è che la Iacova squarquoia non abbia ragione, almeno in parte. La vecchiaia, soprattutto quella femminile, quasi mai è stata vista in chiave positiva ${ }^{5}$. Anzi, viene spesso temuta, perché la sua involuzione fisica viene considerata come qualcosa di diabolico, molto vicino alla stre-

${ }^{5}$ Questo atteggiamento verso le vecchie risulta essere quasi una costante in tutta la storia umana con poche eccezioni. Si ricorda Orazio che nell'Epodo VIII esprime il proprio disgusto verso una vecchia che lo insidia e la cui descrizione sembra anticipare quella basiliana: "Mi chiedi cosa snervi le mie forze tu, / ammuffita da troppo lunga vita, / che hai denti neri, tutta la fronte solcata / di rughe per l'età avanzata / e un culo osceno che si spalanca fra natiche / flaccide di vacca digiuna? / E per eccitarmi mostri sul petto tette / pendule come quelle di cavalla, / un ventre floscio e cosce rinsecchite / sopra polpacci tumefatti..." (testo disponibile sul sito http://www.rodoni.ch/busoni/bibliotechina/orazio/epodi.html). Questo antico disprezzo per la vecchiaia permane anche nelle epoche successive. Nel Medioevo, anche se a prima vista il disprezzo nei confronti della vecchiaia sembra controllato e rivisto attraverso la chiave della religione cristiana, le invettive contro la figura della vecchia sono ricorrenti: Rustico Filippi scrive il sonetto «Dovunque vai con teco porti il cesso, / oi buggeressa vecchia puzzolente» e Franco Sacchetti la ballata «Di diavol vecchia femmina ha natura». La ripugnanza per le vecchie si risveglia con lo stesso antico ardore in epoca rinascimentale quando il culto della bellezza classica ritorna sul piedistallo (Minois 1994). Nell'Elogio della Follia, Erasmo da Rotterdam (2013: 35), fortemente influenzato dalle riflessioni classiche, esprime un giudizio categorico sulla vecchiaia femminile: "Ma nulla c'è di più spassoso di certe vecchie praticamente già morte tanto sono decrepite, $a$ tal punto cadaveriche da sembrare reduci dagl'inferi, ma che hanno sempre sulle labbra il ritornello: "la vita è bella"; fanno ancora le vezzose; mandano sentore di capra - come dicono i Greci; conquistano a caro prezzo un qualche Faone, s'imbellettano di continuo, stanno sempre allo specchio, si sfoltiscono i peli del pube, ostentano le vecchie mammelle avvizzite, sollecitano con tremuli mugolii il desiderio che vien meno, bevono, si inseriscono nelle danze delle fanciulle, scrivono bigliettini amorosi... Vorrei che chi giudica queste cose degne d'irrisione riflettesse un po': è meglio trascorrere nella follia una vita colma di dolcezza, o andare cercando, come suol dirsi, una trave a cui impiccarsi?’”. 
goneria, visto che la decadenza fisica è quasi sempre unita a quella morale. Sulla scia di Aristotele secondo il quale "l'anima è la forma del corpo", Le Goff (2010: 89) spiega anche le conseguenze linguistiche generate da questa avversione verso le vecchie: "Un vocabolo che si incontra frequentemente nelle storie edificanti chiamate exempla, è un segnale di questo atteggiamento ostile: vetula, cioè "vecchietta", che designa invariabilmente un personaggio malvagio".

Il racconto inizia con l'apparizione appunto di due vecchiette, "ch'erano lo reassunto de le desgrazie, lo protacuollo de li scurce [il protocollo delle deformità], lo libro maggiore de la bruttezza" (Basile 2013: 198). Come se questo non bastasse, la fabulatrice Iacova prosegue con una descrizione fisica dettagliata che parte dai loro capelli e finisce con i piedi "a crocco [a uncino]" (Basile 2013: 200). Oltre alla loro bruttezza flagrante, le vecchie, come spesso capita nell'età avanzata, vantano anche un carattere infantile e un comportamento irragionevole, che sarà scambiato da parte del loro vicino di casa, re di Roccaforte in persona, per gentilezza dell'animo. Il re si innamora, ovviamente non per veduta, ma si innamora della voce, cadendo in un altro inganno perché riduce le loro due voci a una voce sola, scambiandola, poi, con "la quantascienza de le cose cenede, lo primmo taglio de le carnumme mellese e l'accoppatura de le tennerumme [la quintessenza delle morbidezze, il primo taglio delle carnucce delicate e il fior fiore delle tenerezze]" (Basile 2013: 200), l'esatto contrario rispetto alla triade descrittiva iniziale. La passione del buon vicino e altrettanto buon conoscitore della lirica cortese diventa smisurata, quasi letteralmente seguendo i dettami di Andrea Cappellano (1996: 15) che dice:

Ma poi che giunge al pensiero pieno, l'amore non sa tenere il freno, e passa subito ai fatti... E comincia a pensare come incontrare la sua grazia, a chiedere luogo e tempo giusto per parlare, e un'ora gli pare un anno, perché non c'è nulla che possa subito saziare l'animo desideroso... Al sorgere dell'amore non basta il semplice pensiero, ma occorre che esso sia smisurato, perché il pensiero misurato non torna insistentemente alla mente, e da lì dunque non può sbocciare amore.

Sin dall'inizio lo scrittore si serve del pensiero del capostipite della tradizione cortese, in chiave palesemente ironica, rovesciando la fin'amor ai propri fini. Al posto della donna amata, bellissima, nobile e per forza anche giovane, il sex symbol basiliano diventa ora una vecchietta alla quale nel trattato di Cappellano (1996: 17) era assolutamente negato il diritto all'amore.

Dedito agli assidui corteggiamenti, il re è diviso tra la brama e la sofferenza amorosa e perseguitato dall'eccessivo appetito sessuale che lo definirà nel corso dell'intero cunto. Il suo comportamento invadente è pieno di allusioni erotiche ispirate alla lirica cortese che di nuovo viene ridicoliz- 
zata perché accostata ai giochi infantili, ora connotati diversamente, in uno spazio riservato esclusivamente agli adulti:

"Dove, dove te nascunne, gioello, sfuorgio, isce bello de lo munno? Iesce, iesce sole, scaglienta 'mparatore! [Dove, dove ti nascondi, gioiello, lusso, cosina bella del mondo? Esci, esci sole, riscalda l'imperatore!] ... apre le porte a povero farcone! Famme la 'nferta si me la vuoi fare! [apri le porte al povero falcone! Dammi la mancia se me la vuoi dare!]” (Basile 2013: 200).

Le vecchie, seguendo la libertà di non impiegare nessuna logica che sarebbe inerente al personaggio o alla situazione, decidono di non rivelarsi al re e nemmeno di avvisarlo dell'equivoco, ma di prendersi la sfida e "de 'ncappare st'auciello che da se stisso se veneva a schiaffare drinto a no codavattolo [... acchiappare un uccello che veniva a ficcarsi da sé dentro la gabbietta]" (Basile 2013: 202). Ma che cosa sta alla base di questa decisione di entrare nel gioco erotico con un re? Le vecchie sono assolutamente consapevoli della propria età, nonché della propria "brutta cera" e di conseguenza anche del fatto che non possono competere con delle ragazze giovani. Però loro le ragazze giovani ovviamente non le prendono nemmeno in considerazione. La loro vita è talmente misera e bassa (anche letteralmente, loro sono " "ncaforchiate [rintanate]", cfr. Basile 2013: 200), che questa proposta indecente da parte del re rappresenta per loro l'ultima possibilità di sentirsi vive, di provare qualche trattenimiento del quale hanno un enorme ed urgente bisogno. Ne hanno necessità loro, come ne hanno i piccirilli. Vogliono vedere uno spettacolo, anzi lo vogliono vivere. In questo modo, l'autore si pone il compito non soltanto di raccontare un cunto, ma di raccontare la propria epoca, che sottoponeva la stessa vita (e anche la morte) a una continua teatralizzazione. Già Benedetto Croce (1924: XXXIII) sottolineava la forma performativa dell'opera basiliana, definendola come "copione destinato al momento del gioco e del riso della corte, un passatempo calibrato sulle regole della conversazione cortigiana". Questa denominazione sarà adottata anche da Michele Rak (2005), che per caratterizzarla userà il termine "cannovaccio". Sul piano del microtesto da noi preso in esame, risulta ovvio il raddoppiamento del pubblico ma anche dei narratori intradiegetici, perché le vecchie recitano lo spettacolo per il re, inventando una nuova identità corporale, il re da parte sua deve anche declamare la propria parte, sapendo che "co li carizze, non co le sbraviate, se "ndorca Venere [con le carezze, non con le bravate, si addolcisce Venere]" (Basile 2013: 204) e tutti questi micro spettacoli rientrano nell'ambito dello "spettacolo degli spettacoli", destinato al pubblico della corte. Così, più che l'invidia, i veri protagonisti dell'opera basiliana, nonché i protagonisti impliciti dei cunti singolari, veri e propri mise em abyme tematici che troveranno il terreno fecondo anche nel cunto in cui le due vecchie diventano l'oggetto libidico di un re, sono il gioco e, il riso e di conseguenza, anche la metamorfosi. 
L'importanza del gioco non trova una giustificazione soltanto intrinseca, nei giochetti erotizzati ed erotici che si scambiano alternativamente le vecchiette e il re, accompagnati da altrettanti giochetti di parole, ma fanno parte integrale dell'opera. I giochi aprono ciascuna delle cinque giornate e insieme ai cunti rappresentano il principale passatempo della compagnia. Eppure, il valore che i narratori danno al gioco va oltre il semplice trattenemiento e presuppone soprattutto un impegno mentale. Là dove c'è gioco, c'è anche la voglia di pensare, di trasgredire, di competere che alla fine può portare soltanto ai vantaggi, come viene spiegato nell'apertura dell'ultima giornata, quando Cola Iacovo, scalco di corte, propone lo iuoco de li iuoche, dicendo che "pocca non sulo se venne a passare lo tiempo co sta manera de iuoche, ma se scetano e fanno prunte li 'nciegne a saperese resorvere e a responnere a chello che se demanna [perché con questo tipo di giochi non solo si passa il tempo, ma si risvegliano e si rendono pronti gli ingegni nel prendere decisioni e nel rispondere a quanto si chiede]" (Basile 2013: 878). Le due vecchiette quindi decidono di giocare e vedremo che quella decisione porterà "guadagno gostuso" (Basile 2013: 878) come promesso da Cola Iacovo. Loro non sono per niente meno fantasiose del re e sembra che anche loro due conoscano bene le convenzioni della letteratura cortese e i suggerimenti di Andrea Cappellano (1996: 127) quando istruisce che "quanto più è difficile scambiarsi piaceri, tanto più cresce la voglia e il desiderio di amare". Consapevoli dell'offuscamento totale della mente del re a causa della sua passione smisurata, approfittano della sua debolezza che non rispecchia per niente il nome del suo principato e promettono di fargli vedere un dito a distanza di otto giorni. Ma è a questo punto che le due vecchiette in effetti pian piano cominciano a perdere la loro posizione vantaggiosa, perché l'incantesimo e il potere indiscutibile della fantasia narrata, personificati anche dalla bambola della protagonista de Lo Cunto de li Cunti, la principessa Zoza, che fila oro e che rappresenta "una bellissima metafora della complessa e raffinata macchina narratologica che si sta proprio ora mettendo in azione personificando il potere della narrazione stessa" (Picone 2003: 305), vengono facilmente annullati di fronte alla realtà fisica. La realtà è un dito decrepito e raggrinzito che non soltanto segnerà 1'approssimarsi della fine del gioco, ma avrà anche un ruolo decisivo per la sorte singolare di ognuna delle sorelle, perché chi tra loro due "avesse lo dito chiù liscio ne facesse mostra a lo re" (Basile 2013: 202) e continuerà il gioco. Vince la sorella "chiù carreca d'anne [la più carica di anni]" (Basile 2013: 204). Per il re di Roccaforte, quel dito che lei ha levigato con la saliva, succhiandolo per otto giorni, aumenterà soltanto il "furore feticistico" e "rappresenta l'epifania dell'inafferabile" (Guaragnella 1986: 545). Il re avvampa ancor di più e la vecchia non ha più nessuna via d'uscita a disposizione e deve rischiare passando al livello successivo del gioco che consiste 
nell'"offerta di tutta la persona" (Cappellano 1996: 26). Gli ultimi attimi di dominio della vecchia consistono nella sua richiesta dell'oscurità totale che sarà interpretata dal re in chiave feticistica e quindi accettata. Così,

\begin{abstract}
"tiratose tutte le rechieppe de la perzona e fattone no rechippo dereto le spalle legato stritto stritto co no capo de spao, se ne venne a la scura, portata pe mano da no cammariero drinto la cammara de lo re, dove, levatose le zandraglie, se schiaffaie drinto a lo lietto [dopo aver stirato tutte le rughe del corpo e averne fatto un nodo dietro le spalle legato stretto con un capo di spago, se ne venne al buio, portata per mano da un servo nella camera del re, dove, tolti gli stracci, si schiaffò nel letto]" (Basile 2013: 206).
\end{abstract}

Fino a questo punto, la vecchia è soltanto una povera "pazzerella", che deve arrangiarsi con i mezzi disponibili e non con la magia o con la stregoneria affinché i segni della sua età avanzata diventino meno visibili e la verità del suo corpo sia trasformata (ovviamente senza successo). Nel buio della stanza reale ogni traccia dell'apparenza vera dell'eroina basiliana è camuffata, il buio nasconde la realtà del suo corpo e l'odore di muschio e zibetto insieme all'esagerata quantità di profumo sul corpo del re annullano "lo schiauro de la vocca soia, l'afeto de le tetelleche e la mofeta de chella brutta cosa [le esalazioni della sua bocca, la puzza delle ascellette e il fetore di quella brutta cosa]" (Basile 2013: 206). Però l'unico senso che non può essere falsato è quello tattile e in effetti sembra che il re si accorga subito della beffa, rendendosi conto "de le caionze secche e de le vessiche mosce ch'erano dereto la poteca de la negra vecchia [della trippa vecchia e delle vesciche sgonfiate che erano dietro la bottega della disgraziata vecchia]" (Basile 2013: 208). Eppure, come osserva Guaragnella (1986: 546), "non è un caso che col tema della sessuofobia si incroci quello di una perversione che quasi sconfina in una forma oscura di masochismo" perché il re non si fa intimidire dalla ripugnanza lampante della vecchia, ma fantasioso com'è, "dette funno a no Mantracchio, mentre se credeva stare a la costa de Posileco e navecaie co na permonara, penzannose de ire ' $n$ curzo co na galera schiorentina [diede fondo in un Mandracchio mentre credeva di stare sulla costa di Posillipo e navigò su una barcaccia pensando di far vela con una galea fiorentina]" (Basile 2013: 208). Per ora, il riso che nasce, provocato dalla goffaggine della vecchia, dall'equivoco creato, dall'erotomania del re, ecc. è un riso che viene destinato al pubblico "esterno". Ma come succede con il gioco, anche il riso, come vedremo, sarà moltiplicato e rivalorizzato all'interno dello stesso cunto.

Quando la vecchietta cade in un sonno profondo, il re accende una lucerna con la quale si accerta di quello che intimamente già sapeva. Sino a questo momento non c'è nessun incantesimo, nessuna magia, c'è soltanto un equivoco dalle dimensioni fatali che nasconde, però, un'altra spia fondamentale che preannuncia la metamorfosi finale: la storia di Psiche 
e Amore. Italo Calvino (2001: XLVIII) sosteneva che il mito di Psiche e Amore era dominante nelle fiabe e nei racconti popolari italiani. In Lo Cunto de li Cunti, il Basile legge il mito apuliano in chiave barocca, ovvero alla rovescia, negando la presenza della provvidenza che porta all'autorealizzazione e quindi rifiutando le interpretazioni tipiche delle epoche precedenti che assegnavano all'interpretazione del mito una dominante dimensione religiosa. Il Basile si avvale di alcuni motivi del mito di Psiche e Amore che a volte funzionano come delle "unità narrative indipendenti"' (Maggi 2015: 34): il diniego di vedere la faccia dell'amante notturno/a e poi la rottura di questa proibizione, la scoperta della vera identità, l'umiliazione e l'allontanamento, il matrimonio, ecc., e poi le rimodella, cambiando a volte anche i ruoli di Psiche e di Amore e riconfermando sì che la rivelazione conseguita alla fine della ricerca dell'anima è il risveglio, ma un risveglio in mezzo all'orrore quotidiano (Maggi 2015: 34-35). L'orrore riduce Arpia, Furia e Gorgone a un corpo solo che, nonostante le lamentele e il ricorso a proverbi stereotipati come "gallina vecchia fa buono bruodo" e "non se deve lassare la via vecchia pe la nova" (Basile 2013: 208), viene crudelmente buttato dalla finestra, ma fortunatamente salvato da un albero.

A questo punto inizia la seconda parte, decisiva in quanto per la prima volta appaiono improvvisamente delle figure magiche, in veste di "certe fate... le quale pe na certa crepantiglia [per una certa stizza] non avevano mai parlato né riso" (Basile 2013: 210) che passando sotto l'albero dal quale pendeva la vecchia, alla vista di una scena talmente bizzarra, scoppiano a ridere. Grate per quell'inaspettato dono della risata, le fate decidono di premiare la vecchia donandole gioventù, bellezza, ricchezza, nobiltà, virtù, amabilità e fortuna. Nasce quindi una bellezza suprema, che si trova in antitesi con la descrizione iniziale della sua predecessora e sembra l'ispirazione anticipata della descrizione di Croce (1924: XIII) del Seicento: "Età di decadenza, sia pure; ma importa non dimenticare che il concetto di decadenza è affatto empirico e relativo: se qualcosa decade, qualche altra cosa nasce e germina: una decadenza totale e assoluta non è concepibile." Il corpo raccoglie in sé tutto il repertorio della bellezza classica, dalla carnagione, dai capelli fino al decoro esterno, e non rivela nessuna traccia della sua precedente proprietaria, ma si mostra nella piena bellezza di una quindicenne (dall'anima vetusta), così il re in un istante dimentica la scena precedente, pronto per un altro gioco erotico che stavolta finisce con la festa di nozze.

In questo modo, il riso alla pari del gioco che fu all'origine dell'intero processo, inaspettatamente porterà un "guadagno" decisivo, cambiando il destino della protagonista. Il dono delle fate consiste in una metamorfosi radicale, preannunciata vagamente nella scena di Psiche e Amore, che investe

\footnotetext{
${ }^{6}$ Tutte le citazioni tratte da Maggi (2015) sono date in traduzione dall'inglese.
} 
sia il corpo della vecchietta che il suo status sociale, perché soltanto quando entrambi i requisiti saranno soddisfatti, la vecchia renovata può diventare la degna sposa del re. E tutto questo grazie al riso, che non a caso, diventa il motivo intertestuale ${ }^{7}$ per eccellenza ed entra in confronto con il macrotesto e non soltanto. Inserendo la questione del riso nella struttura intrinseca dell'opera, il Basile fa risorgere l'antica problematica del riso, tramite la quale offre la sua visione del mondo, rivedendolo ora dalla prospettiva della sua epoca. Il riso, che una volta era visto soprattutto come la "lordura della bocca" (Le Goff 2010: 61-62), siccome proveniva dal ventre, la parte ignobile della topografia del corpo, connotata negativamente (Le Goff 2010: 61), nel Basile assume non tanto una connotazione per forza positiva, quanto il carattere di una potenza rigenerativa che produce "svolte definitive grazie alle quali i percorsi diventano destini" (Rak 2013: XXXIV). Di fronte alle donne malinconiche decameroniane, il Basile pone la malinconia della principessa Zoza che apre l'intera opera, solo che a differenza del Decameron, il cunto proemiale basiliano è "un testo narrativo [...] è la novella fondamentale che giustifica tutte le altre novelle che vengono affabulate al suo interno" (Picone 2003: 299). L'incapacità di Zoza di ridere è legata alla sua immaturità sessuale. Il suo umore dominante trasmuterà grazie all'oscenità, questa volta di un'altra vecchia, che mostrerà impudicamente il proprio sesso davanti agli occhi della ragazza. La vista dei genitali della vecchia implica la scoperta e la coscienza intima della propria mancanza e il riso che ne consegue, arriva soprattutto come una reazione liberatoria, segnando l'inizio di un lungo percorso verso la maturità e la sua iniziazione emotiva e sessuale. Così, la malinconia all'interno dell'opera basiliana assume un carattere doppio: o si presenta come "incapacità letargica di vivere e di ridere" o come "frenetico ed estenuante impulso verso l'azione" (Maggi 2015: 93). D'altra parte, la potenza del riso nel Basile viene equiparata alla potenza del parlare/raccontare e quindi all'attuazione della persona, siccome si tratta di qualcosa di inerente esclusivamente alla natura umana. Così come il riso è una prerogativa dell'uomo (Le Goff 2010: 60), secondo il principe Tadeo "il racconto è una predisposizione originaria dell'uomo" (Picone 2003: 309), "l'utema felicità dell'ommo" (Basile 2013: 22). E infatti, le fate del cunto "La Vecchia scorticata" non hanno mai parlato e, di conseguenza, non hanno mai riso e la malinconia della principessa Zoza, e poi l'omore malanconeco della moglie-schiava generano la narrazione,

${ }^{7}$ Pur essendo definito marinista mediocre, con il suo approccio intertestuale e interdiscorsivo di natura altamente polemica, il Basile si accosta a Marino che considerava un'opera letteraria come un insieme di testi già esistenti, integrati ora in un contesto nuovo, grazie al quale l'opera assume anche significati nuovi (Zogović 2007: 73-82). 
l'antidoto contro la malinconia. Solo grazie all'enorme forza del racconto e del riso, la metamorfosi diventa possibile.

Attraverso il gioco, la malinconia, il riso e la metamorfosi, il Basile offre la sua visione della vita e del tempo, visti non più come qualcosa di rigido, dotato di un proprio inizio e di una propria fine definitiva, ma come qualcosa di instabile, un processo eterno che cambia soltanto la forma e trabocca da un'immagine all'altra. Le concezioni del gioco e del riso non sono soltanto fondamentali per la comprensione dei cunti basiliani, ma la sua impostazione dell'intero sistema narrativo è ludica. Utilizzando certi motivi, caratteri o ciò che Propp (2000) chiama "funzioni" che potrebbero essere definiti "fiabeschi", il Basile indirizza il suo interesse esclusivamente verso la realtà, creando una scrittura iperrealistica a cui tutti gli elementi partecipano unanimemente per esprimere e interpretare quella realtà: la lingua trasmette la sua fluidità e incostanza ${ }^{8}$ cancellando i confini precisi tra l'apparenza e la realtà, mentre invece l'intreccio del cunto enfatizza che le certezze sono sparite, che tutto diventa relativo e pluriforme, come anche la sorte della sorella più fortunata. La visione pessimistica della vita si trasforma nell'assenza di regole che eliminino la possibilità di un lieto fine: non sappiamo se il premio delle fate durerà per sempre o svanirà nel nulla improvvisamente così come era venuto, come ci viene appunto suggerito dall'egloga che chiude la prima giornata "La coppella":

Quanto a la 'ncornatura e a primma fronte

pare cosa de priezzo,

tutto "nganna la vista,

tutto ceca la gente,

tutto è schitto apparenzia.

(Basile 2013: 224, 226)

[Tutto quello che dalla testa e dalla facciata / sembra roba di valore, / è tutto un inganno della vista, / è tutto un velo sugli occhi della gente, / è tutto soltanto apparenza].

${ }^{8}$ Quello che Getto (2000: 310-311) definisce come "incontentabilità della parola", precisando più in avanti che "attraverso questo linguaggio il Basile esprime la sua visione del reale, irrequieto e sfuggente, molteplice e problematico, tumultuoso e inesauribile. La realtà acquista come un senso turgido e traboccante" (Getto 2000: 313). 


\section{BIBLIOGRAFIA}

Basile, G. (2013). Lo Cunto de li Cunti overo Lo Trattenemiento de Peccerille de Gian Alessio Abbattutis, a cura di M. Rak. Milano: Garzanti.

Calvino, I. (2001). Introduzione di Italo Calvino. In Fiabe italiane, raccolte dalla tradizione popolare durante gli ultimi cento anni e trascritte in lingua dai vari dialetti da Italo Calvino (pp. VII-LIV). Milano: Arnoldo Mondadori.

Cappellano, A. (1996). De Amore, traduzione di J. Insana. Milano: SE.

Croce, B. (1924). Saggi sulla letteratura italiana del Seicento. Bari: Laterza.

Erasmo da Rotterdam (2013). Elogio alla Follia. Raleigh: Aonia edizioni.

Getto, G. (2000). La fiaba di Giambattista Basile. In Il Barocco letterario in Italia (pp. 296-313). Milano: Bruno Mondadori.

Guaragnella, P. (1986). Eros vecchiezza metamorfosi. Su una fiaba di G. B. Basile. Lares, 52, 4, 535-552.

Le Goff, J. (2010). Il corpo nel Medioevo. Bari: Laterza.

Maggi, A. (2015). Preserving the Spell. Basile's "The Tale of Tales" and Its Afterlife in the Fairy-Tale Tradition. Chicago: The University of Chicago Press.

Minois, G. (1988). Storia della Vecchiaia dall'Antichità al Rinascimento. Bari: Laterza.

Picone, M. (2003). La Cornice Novellistica dal Decameron al Pentamerone. Modern Philology, 101, 2, 297-315.

Propp, V. (2000). Morfologia della fiaba. Torino: Einaudi.

Rak, M. (2005). Logica della fiaba. Milano: Bruno Mondadori.

Rak, M. (2013). Il racconto fiabesco. In G. Basile (a cura di M. Rak), Lo Cunto de li Cunti overo Lo Trattenemiento de Peccerille de Gian Alessio Abbattutis (pp. XXXII-LXXI). Milano: Garzanti.

Zogović, M. (2007). Barok: književna teorija i praksa. Beograd: Narodna knjiga Alfa.

Sitografia

https://books.google.rs/books?id=P75BOzIsooEC\&printsec $=$ frontcover\& $\mathrm{hl}=\mathrm{it} \# \mathrm{v}=$ onepage $\& \mathrm{q} \& \mathrm{f}=$ false

http://www.rodoni.ch/busoni/bibliotechina/orazio/epodi.html 


\section{SEXUALITY AND SENESCENCE IN ONE BASILE'S CUNTO}

\section{Summary}

In the Baroque era, when the primary artistic assignment becomes meraviglia (marvel) and when the artists explore inside the realities marginalized during the previous epochs, the artists' attraction by the body identity originates some themes and characters almost non-existent until then. Thus, Basile in one tale of his monumental work The Tale of Tales introduces two old women, as the protagonists, who become an object of a sexual desire of a king. With this unusual plot, the writer not only satisfies the oddities of Baroque and its thematic and stylistic imperatives, but also opens some very complex questions with which he polemicizes with previous epochs, offering above all, his own vision of life, this time in a relative, pessimistic and multiform perspective.

Keywords: Basile, Baroque, old age, game, laugh, metamorphosis. 